\title{
Study of Micronutrients and Dyslipidemia in T2DM
}

\author{
${ }^{1}$ Associate Professor, Department of Biochemistry, SSMC, Tumkur, India \\ ${ }^{2}$ Assistant Professor, Department of Biochemistry, MMC\&RI, Mysore, India \\ ${ }^{3}$ Associate Professor, Department of Physiology, SSMC, Tumkur, India \\ ${ }^{4}$ Assistant Professor, Department of Physiology, SSMC, Tumkur, India
}

Sathisha T. $\mathrm{G}^{1}$, Rangaswamy $\mathrm{R}^{2^{*}}$, Bhargavi S. $\mathrm{K}^{1}$, Shashiraj H. $\mathrm{K}^{3}$, Kavitha B. $\mathrm{S}^{4}$, Arifuddin M. $\mathrm{K}^{4}$

DOI: $10.36348 /$ sijb.2020.v03i07.001

| Received: 19.06.2020 | Accepted: 26.06.2020 | Published: 05.07.2020

*Corresponding author: Dr. Rangaswamy R

Abstract

Type 2 Diabetes Mellitus (T2DM) is a chronic metabolic disorder because of absolute or relative deficiency of insulin production by the beta cells of the pancreas. Trace elements like Zinc and copper are known to affect the synthesis, secretion, release and mechanism of action of insulin. Dyslipidemia is an associated abnormality in most of T2DM patients and the underlying mechanism is not resolved well. Estimation of the trace elements and lipid profile in newly diagnosed T2DM patients will help to identify the onset of early oxidative damage and prevent various complications of T2DM. The present case control study incorporated age and sex matched 48 type 2 diabetic patients and 48 apparently healthy adults. Copper was estimated by Di-Br-PAESA method and Zinc by Nitro PAPS method on Chem 7. HDL-C and TGL quantification was done by EM200 Fully auto analyzer by respective kits from manufacturer. The present study showed that trace elements like serum Copper levels were significantly increased $(\mathrm{P}<0.001)$ where as serum Zinc levels were significantly decreased $(\mathrm{P}<0.001)$. At the same time HDL-C was significantly decreased $(\mathrm{P}<0.001)$ and TGL significantly increased $(\mathrm{P}<0.001)$ in diabetic patients in comparison to controls. Abnormalities in trace elements metabolism and deranged lipid profile may add to the increased risk of complications in T2DM. Early estimation of trace elements and supplementation of the same with therapeutic intervention and correction of lipid abnormalities will help to improve the treatment outcome in these patients.

Keywords: T2DM, Copper, Zinc, HDL-C, Triglycerides, Dyslipidemia.

Copyright @ 2020: This is an open-access article distributed under the terms of the Creative Commons Attribution license which permits unrestricted use, distribution, and reproduction in any medium for non-commercial use (NonCommercial, or CC-BY-NC) provided the original author and source are credited.

\section{INTRODUCTION}

Type 2 Diabetes Mellitus (T2DM) is a chronic metabolic disorder because of absolute or relative deficiency of insulin production by the beta cells of the pancreas. Impaired insulin secretion affects carbohydrate, protein and lipid metabolism and poorly controlled blood glucose levels can lead to various complications of diabetes like nephropathy, neuropathy and retinopathy $[1,2]$. It is estimated that 415 million people are living with diabetes in the world which is estimated to be one in 11 of the world's adult population. The figure is expected to rise to 642 million people living with diabetes worldwide by 2040 . Diabetes currently affects more than 62 million Indians which are more than $7.2 \%$ of the adult population [3].

Hyperglycemia in T2DM is known to lead to over production of free radicals and ROS. Oxidative stress, which has been implicated in the etiopathogenesis of T2DM leads to increased formation of advanced glycation end (AGE) product by non enzymatic glycation of proteins. Accumulation of AGEs can cause endothelial damage which explains the vascular complications of DM leading to increased morbidity and mortality $[4,5]$.

Trace elements like Zinc and copper are known to affect the synthesis, secretion, release and mechanism of action of insulin [1, 6]. Dyslipidemia is an associated abnormality in most of T2DM patients and the underlying mechanism is not resolved well [7]. So it becomes important to estimate the trace elements and lipid profile in newly diagnosed T2DM patients to prevent early oxidative damage which might later lead to various complications of T2DM.

The present study was undertaken to assess the association between trace elements and lipid profile of T2DM patients among patients attending the outpatient department of SSMC Hospital, Tumkur. 


\section{MATERIALS AND METHODS}

The present case control study incorporated age and sex matched 48 type 2 diabetic patients and 48 apparently healthy adults. SSMC Hospital, Tumkur provided the diabetic patients. Controls were healthy adults indiscriminately selected. 2019-2020 was the time frame of study. The history, physical examination and biochemical investigations were the sources of diagnosis. Ethical clearance was approved for the study by the institutional ethics committee on human experimentation in accordance with the revised Helsinki Declaration. Informed consent was signed by each subject.

\section{Inclusion Criteria}

Newly diagnosed Diabetic Patients and controls in the age group 40-60 years.

\section{Exclusion Criteria}

Patients or controls suffering from acute metabolic complications such as hypoglycemia, ketoacidosis, cerebrovascular accident, acute infection, primary hypertensives, smokers and alcoholics. Patients who had already received or were under treatment for diabetes were excluded from the study. Clinical investigations and questionnaires formed the basis of enquiry.

\section{Collection of Blood}

$5 \mathrm{ml}$ of fasting venous blood was drawn from median cubital/basilic vein under strict aseptic precautions into BD red capped plain vacutainers. They were allowed to stand at room temperature for 10 minutes for clotting to occur. Soon after they were centrifuged at 3000rpm for 10 minutes using Remi8RC centrifuge. Serum was separated immediately. All the parameters were estimated on same day.
Metal ions were assayed by UV- Visible Spectrophotometer- CHEM 7 using Tulip diagnostics (P) Ltd kits.

\section{Copper Di-Br-PAESA method}

Copper is released in acidic medium from ceruloplasmin. Free copper reacts with Di-Br-PAESA to form a colored complex. The concentration of Copper present in the sample is directly proportional to intensity of colored complex measured at wavelength $\mathrm{Hg} 578 \mathrm{~nm}$.

\section{Zinc Nitro PAPS method}

Zinc in alkaline medium reacts with NitroPAPS to form a purple colored complex. Intensity of colored complex is directly proportional to the concentration of Zinc present in the sample measured at wavelength $\mathrm{Hg} 578 \mathrm{~nm}$

HDL-C and TGL were estimated by EM 200 fully auto analyzer by respective kits from manufacturer. Quality control of results was ensured by running normal and abnormal reference control serum solutions daily. Manufacturer's recommendations like initial calibration and routine maintenance were fallowed.

\section{Statistical Analysis}

Excel spread sheet was utilized to enter the data. Data cleaning and validation was completed. The study data was analyzed using Statistical Package for Social Sciences (SPSS-Version 20) (IBM Corp., Armonk, NY, USA). Kolmogorov-Smirnov test was utilized for testing all the variables for normality. Data was presented as Mean and Standard deviation for continuous variables. Variables were compared using an independent sample t-test for normally distributed data. P-value $<0.05$ was considered statistically significant.

\section{RESULTS}

Table-1: Comparison of Copper, Zinc, HDL- Cholesterol and Triglycerides between control and diabetic patients

\begin{tabular}{|l|l|l|l|l|l|}
\hline Parameter & Group & N & Mean & SD & P-value \\
\hline \multirow{2}{*}{$\begin{array}{l}\text { Copper } \\
(\mu \mathrm{g} / \mathrm{dL})\end{array}$} & Control & 48 & 112.36 & 13.72 & $<0.001$ (Significant) \\
\cline { 2 - 5 } & Cases & 48 & 179.62 & 19.67 & \\
\hline $\begin{array}{l}\text { Zinc } \\
(\mu \mathrm{g} / \mathrm{dL})\end{array}$ & Control & 48 & 86.14 & 9.25 & $<0.001$ (Significant) \\
\cline { 2 - 5 } & Cases & 48 & 42.85 & 6.34 & \\
\hline $\begin{array}{l}\text { HDL- C } \\
(\mathrm{mg} / \mathrm{dl})\end{array}$ & Control & 48 & 48.29 & 5.37 & \multirow{2}{*}{$<0.001$ (Significant) } \\
\cline { 2 - 5 } & Cases & 48 & 35.16 & 3.28 & \\
\hline \multirow{2}{*}{$\begin{array}{l}\text { Triglycerides } \\
(\mathrm{mg} / \mathrm{dl})\end{array}$} & Control & 48 & 118.47 & 9.52 & $<0.001$ (Significant) \\
\cline { 2 - 5 } & Cases & 48 & 162.53 & 14.45 & \\
\hline
\end{tabular}




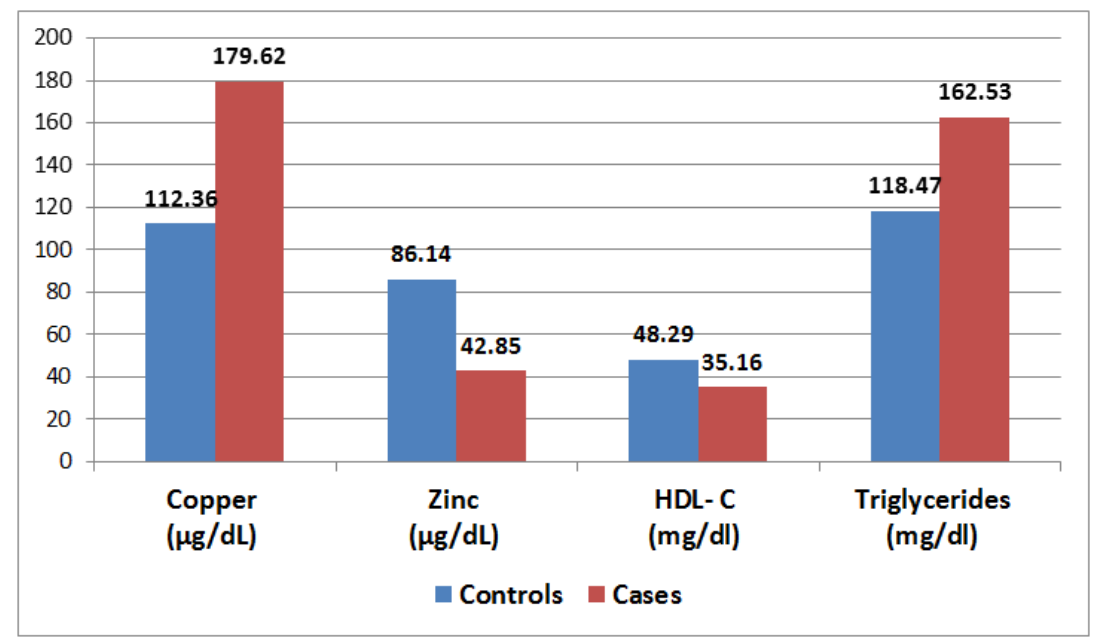

Graph-1: Comparison of Copper, Zinc, HDL- Cholesterol and Triglycerides Between control and diabetic patients

The trace elements like serum Copper levels were significantly increased $(\mathrm{P}<0.001)$ where as serum Zinc levels were significantly decreased $(\mathrm{P}<0.001)$ in diabetic patients in comparison to controls. At the same time HDL-C is significantly decreased $(\mathrm{P}<0.001)$ and TGL is significantly increased $(\mathrm{P}<0.001)$ in diabetic patients in comparison to controls.

All the parameters assayed were significantly altered $(\mathrm{P}<0.001)$ between controls and diabetics.

\section{DISCUSSION}

Diabetes Mellitus is a globally affecting debilitating chronic disease. Globalization implicates the people of developing countries more into the sedentary life because of urbanization. Sedentary life style perpetuates into T2DM.

Copper is essential for the catalytic activity of superoxide dismutase (SOD) along with Zinc. SOD functions by scavenging superoxide radicals there by guarding the cells from oxidative damage [8]. SOD catalyses dismutation of super oxide anion into hydrogen peroxide and oxygen. Out of three isoforms in mammals, isoform 1 (CuZnSOD) requires copper. Error in copper metabolism ends in lowering the guard on cells from superoxide radicals [9].

Cytochrome oxidase of electron transport chain is activated by copper. Abnormality of copper levels in biofluids is drawn into the aberrations of metabolic pathways of diabetes [10]. This study yielded statistically significant $(\mathrm{P}<0.001)$ high levels of serum copper in diabetic patients with mean \pm standard deviation of $179.62 \pm 19.67$ as compared to controls with mean \pm standard deviation of $112.36 \pm 13.72$. Our study showed significantly elevated serum copper levels in diabetics in contrast to controls which correlates to earlier studies $[11,12]$.
Zinc acts a cofactor for Super Oxide Dismutase SOD dismutase and inhibits NADPHoxidase. Its mammalian isoforms 1 and 3 need Zinc as cofactors to function in both intra as well as extracellular fluids. Zinc controls the expression of glutamate-cysteine ligase implicated in the synthesis of glutathione by acting as a cofactor of glutathione peroxidase as well as the neutralization of free radicals. Zinc competes with copper and iron for binding sites in cell membrane. These ions mediate synthesis of lipid peroxides. Lipid peroxidation can be curtailed if Zinc is swapped for copper and iron. Above mentioned are the modes of Zinc as an antioxidant [13].

Secretion and storage of insulin are mainly regulated by Zinc. It also enhances the uptake of glucose. The key protein for the regulation of insulin is $\mathrm{Zn}$ transporter (ZnT8), a secretion of the pancreatic $\beta$ cells. A mutation in ZnT8 transporter has been associated with T2DM [14].

Hyperglycemia is the constant feature of T2DM which leads to osmotic diuresis. This diuresis is the impending cause for the altered trace elements in the blood of diabetic patient [15]. Serum Zinc levels are less in T2DM patients because increased loss of Zinc in urine. This leads to hypozincemia and hyperzincuria in diabetic patients, as hyperglycemia interferes in active transport of Zinc into renal tubular cells [16, 17].

The low levels of Zinc hamper the functioning of islet cells to produce and secrete insulin [18]. The present study revealed statistically significant $(\mathrm{P}<$ 0.001) low levels of serum Zinc in diabetic patients with mean \pm standard deviation of $42.85 \pm 6.34$ as compared to controls with mean \pm standard deviation of $86.14 \pm 9.25$. Hence the study results of low serum zinc levels in diabetic patients in our case are in accordance with above studies. 
One of the classic characteristic of Type 2 DM is dyslipidemia. It is an indicator of altered lipid metabolism inside the body. It may be attributed by insulin resistance in adipose tissue coupled with hampered insulin secretion. Hepatocytes synthesize more glucose in addition to high flux of free fatty acids from adipocytes. These effect into elevated lipid production [19].

Zinc plays a prominent role in carbohydrate metabolism and insulin actions. Secretion and storage of insulin are mainly regulated by Zinc. It also enhances the uptake of glucose into cells by phosphorylation of insulin receptors. Zinc is the catalytic cofactor for carboxypeptidase $\mathrm{H}$ enzyme which catalyzes the conversion from proinsulin (inactive) into insulin (active). The low levels of Zinc hamper the functioning of islet cells to produce and secrete insulin [20].

The pathophysiology behind diabetic dyslipidemia is enhanced functioning of proprotein convertase subtilisin/Kexin 9 (PCSK9) and cholesteryl ester transfer protein (CETP), less activity of lipoprotein lipase along with damaged lipoprotein clearance. These were recognized as imminent factors for dyslipidemia [21]. Copper disparity causes disturbed normal high density lipoproteins (HDL) and low density lipoproteins (LDL) balance [22]. Current study yields decreased levels of HDL in diabetic patients with mean \pm standard deviation of $35.16 \pm 3.28$ as compared to controls with mean \pm standard deviation of $48.29 \pm$ 5.37. High levels TGL in diabetic patients with mean \pm standard deviation of $162.53 \pm 14.45$ as compared to controls with mean \pm standard deviation of 118.47 \pm 9.52. As observed in present study, the results are consistent with previous studies.

\section{CONCLUSION}

Non enzymatic glycation and oxidative stress associated with T2DM profoundly increases the incidence of vascular complications of DM. Abnormalities in trace element metabolism and the deranged lipid profile may add to the increased risk of complications in T2DM. Early estimation of trace elements and supplementation of the same with therapeutic intervention and correction of lipid abnormalities will help to improve the treatment outcome in these patients. It is recommended to estimate other trace elements like Magnesium, Calcium and more in a large group multi centric study to objectively assess the role of these trace elements in T2DM.

\section{REFERENCES}

1. Praveeena, S., PaSula, S., \& Sameera, K. (2013). Trace elements in diabetes mellitus. Journal of Clinical and Diagnostic Research: JCDR, 7(9), 1863-1865.

2. Center for Disease Control and Prevention. (2014). National diabetes statistics report: estimates of diabetes and its burden in the United States, 2014. Atlanta (GA): US Department of Health and Human Services.

3. Diabetes Prevalence. (2019). Internet Retrieved on June 20, 2020, from www.diabetes.co.uk

4. Cheeseman, K. H., \& Slater, T. F. (1993). An introduction to free radical biochemistry. British medical bulletin, 49(3), 481-493.

5. Wolff, S. P., Jiang, Z. Y., \& Hunt, J. V. (1991). Protein glycation and oxidative stress in diabetes mellitus and ageing. Free Radical Biology and Medicine, 10(5), 339-352.

6. Ibrahim, A., Nasrat, W., \& Elhefian, E. A. (2016). Evaluation of biochemical parameters and trace elements in Type-2 diabetic patients. Nova, 5(4), 1-7.

7. Arca, M., Pigna, G., \& Favoccia, C. (2012). Mechanisms of diabetic dyslipidemia: relevance for atherogenesis. Current vascular pharmacology, 10(6), 684-686.

8. Olivares, M., Araya, M., \& Uauy, R. (2000). Copper homeostasis in infant nutrition: deficit and excess. Journal of pediatric gastroenterology and nutrition, 31(2), 102-111.

9. Di Naso, F. C., Dias, A. S., Porawski, M., \& Marroni, N. A. P. (2011). Exogenous superoxide dismutase: action on liver oxidative stress in animals with streptozotocin-induced diabetes. Experimental Diabetes Research, 2011.

10. Eaton, J. W., \& Qian, M. (2002). Interactions of copper with glycated proteins: possible involvement in the etiology of diabetic neuropathy. Molecular and cellular biochemistry, 234(1), 135-142.

11. Abou-Seif, M. A., \& Youssef, A. A. (2004). Evaluation of some biochemical changes in diabetic patients. Clinica Chimica Acta,346(2), 161-170.

12. Zhao, C., Wang, H., Zhang, J., \& Feng, L. (2008). Correlations of trace elements, glucose and body compositions in type 2 diabetics. Wei sheng yan jiu= Journal of hygiene research, 37(5), 600-1.

13. Oteiza, P. I. (2012). Zinc and the modulation of redox homeostasis. Free Radical Biology and Medicine, 53(9), 1748-1759.

14. Wijesekara, N., Dai, F. F., Hardy, A. B., Giglou, P. R., Bhattacharjee, A., Koshkin, V., ... \& Wheeler, M. B. (2010). Beta cell-specific Znt8 deletion in mice causes marked defects in insulin processing, crystallisation and secretion. Diabetologia, 53(8), 1656-1668.

15. Viktorínová, A., Tošerová, E., Križko, M., \& Duračková, Z. (2009). Altered metabolism of copper, zinc, and magnesium is associated with increased levels of glycated hemoglobin in patients with diabetes mellitus. Metabolism, 58(10), 1477-1482.

16. Kazi, T. G., Afridi, H. I., Kazi, N., Jamali, M. K., Arain, M. B., Jalbani, N., \& Kandhro, G. A. 
(2008). Copper, chromium, manganese, iron, nickel, and zinc levels in biological samples of diabetes mellitus patients. Biological trace element research, 122(1), 1-18.

17. Forte, G., Bocca, B., Peruzzu, A., Tolu, F., Asara, Y., Farace, C., ... \& Madeddu, R. (2013). Blood metals concentration in type 1 and type 2 diabetics. Biological trace element research, 156(1-3), 79-90.

18. Brender, J. R., Hartman, K., Nanga, R. P. R., Popovych, N., de la Salud Bea, R., Vivekanandan, S., ... \& Ramamoorthy, A. (2010). Role of zinc in human islet amyloid polypeptide aggregation. Journal of the American Chemical Society, 132(26), 8973-8983.

19. Powers, A. C. Diabetes mellitus. In: Longo, D. L., Fauci, A. S., Kasper, D. L., Hauser, S. L., Jameson, J., \& Loscalzo, J., editors. (2012)
Harrison's Principle of Internal Medicine. 18th ed. New York, McGraw Hill, 2968-9.

20. Miao, X., Sun, W., Fu, Y., Miao, L., \& Cai, L. (2013). Zinc homeostasis in the metabolic syndrome and diabetes. Frontiers of medicine, 7(1), 31-52.

21. Girona, J., Ibarretxe, D., Plana, N., GuaitaEsteruelas, S., Amigo, N., Heras, M., \& Masana, L. (2016). Circulating PCSK9 levels and CETP plasma activity are independently associated in patients with metabolic diseases. Cardiovascular diabetology, 15(1), 107-112.

22. Jackson, R. F., Roche, G. C., \& Wisler, K. (2010). Reduction in cholesterol and triglyceride serum levels following low-level laser irradiation: a noncontrolled, nonrandomized pilot study. American Journal of Cosmetic Surgery, 27(4), 177-184. 